\title{
Why Are Risk-Pooling and Risk-Sharing Arrangements Necessary for Financing Healthcare and Improving Health Outcomes in Low and Lower Middle-Income Countries
}

\author{
Ali Ahangar', Ali Mohammad Ahmadi² ${ }^{*}$, Amir Hossein Mozayani², Sajjad Faraji Dizaji ${ }^{3}$ \\ ${ }^{1}$ School of Economics and Management, Tarbiat Modarres University (TMU), Tehran, Iran \\ ${ }^{2}$ Economic Research Center (ERC), Tarbiat Modarres University (TMU), Tehran, Iran \\ ${ }^{3}$ Department of Economic Development and Planning, School of Economics and Management, Tarbiat Modarres University \\ (TMU), Tehran, Iran \\ Email: ali_ahangar51@yahoo.com, ^aahmadi@modares.ac.ir, mozayani@modares.ac.ir, s_dizaji@modares.ac.ir
}

How to cite this paper: Ahangar, A., Ahmadi, A.M., Mozayani, A.H. and Faraji Dizaji, S. (2018) Why Are Risk-Pooling and Risk-Sharing Arrangements Necessary for Financing Healthcare and Improving Health Outcomes in Low and Lower Middle-Income Countries. Health, 10, 122-131.

https://doi.org/10.4236/health.2018.101010

Received: December 2, 2017

Accepted: January 23, 2018

Published: January 26, 2018

Copyright $\odot 2018$ by authors and Scientific Research Publishing Inc. This work is licensed under the Creative Commons Attribution International License (CC BY 4.0).

http://creativecommons.org/licenses/by/4.0/ Open Access

\begin{abstract}
Health is important to economic development, and economic development has an important impact on health outcomes. Health Expenditure makes up a substantial part of the global economy. In the world, the costs of healthcare are increasing; patients are compelled to pay more for treatment, and that makes a lot of people faced to Catastrophic Health Expenditures (CHE) and in long run fall below the poverty line. One of the most urgent and vexing challenges faced by many low- and middle-income countries is how to provide health care for the more than two billion poor people who live in these areas (developing countries). As much as more than 65\% (in 2014) of total private health care expenditure in low-income countries comes from out-of-pocket payment by patients. In addition, according to World Bank report (2007), in low and lower middle-income countries was speared nearly $13 \%$ of global health spending with $87 \%$ the global disease burden. The WHO considers health financing models with high risk pooled, such as health insurance and prepaid schemes, a promising means for achieving universal health-care coverage and promotion health care. A crucial concept in health financing is that of pooling. The WHO defines risk-pooling as the "accumulation and management of revenues in such a way as to ensure that the risk of having to pay for health care is borne by all members of the pool and not by each contributor individually". The larger degree of pooling, the less people will have to bear the health financial risks. Furthermore, adopting and operating financing
\end{abstract}


policies based on greeter risk pooling/sharing (prepayments) are recommended to all countries (especially in low and lower-middle income countries). It means risk sharing/pooling plays a key role in all financing systems for achieving effectiveness and efficiency health systems.

\section{Keywords}

Risk Pooling, Risk Sharing, Health Care Financing, Health Economics, Low and Middle Income

Health is important to economic development, and economic development has an important impact on health outcomes. Health Expenditure makes up a substantial part of the global economy. In the world, the healthcare expenditures are strongly increasing; many people are compelled to pay more for treatment, and that makes a lot of people faced to Catastrophic Health Expenditures (CHE) and in long run fall to below the poverty line [1]. One of the most urgent and vexing challenges faced by many low- and middle-income countries is how to provide health care for the more than two billion poor people who live in these areas. The share of resources allocated worldwide to health is increasing faster than ever. One of the main strategies for the government to contain health care costs is higher patient cost sharing, that is, requiring patients to pay a larger share of the cost of care. While cost sharing may reduce direct costs by decreasing the moral hazard of health care services, it may also reduce access to beneficial and necessary health care that could mitigate future severe and costly health events [2]. As much as 80 percent of total health care expenditure in low-income countries comes from direct out-of-pocket payment by patients. Studies in several countries found that large medical expenditure is a major cause of poverty [1].

In addition, according to World Bank Report (2004), that's estimated only $11 \%$ of Global Health Spending for 90\% of the World's Population in developing countries such as Asia 3.5\%, Americas 3.2\%, Europe 2.4\%, Middle East and N. Africa $1.5 \%$, Africa $0.4 \%$, and $89 \%$ of Global Health Spending for $10 \%$ World's Population in developed countries [3]. In 2007 with respect to the distribution of the global disease burden in low- and middle-income countries $87.5 \%$, but only 12.5 percent of global health spending was in this group of countries. Conversely, in developed and highly developed countries, with a very low distribution of global disease burden (13\%), the share of total health expenditures is much higher at $87 \%$ [1]. The World Health Report (2013) stated that inefficiency of the health department financing system has led to a waste of about $20 \%$ to $40 \%$ of the total health expenditures. Therefore, it counts the need to adopt proper and efficient financing policies based on risk pooling and risk sharing in the health sector as an evident issue [1].

The World Health Organization considers health financing models with high risk pooled, such as health insurance and prepaid schemes, a promising means for achieving universal health-care coverage [4]. Risk-sharing in health financing 
is defined as "Any system which allows payors to share some of the financial risk associated with a particular patient population with providers. Providers agree to adhere to fixed fee schedules in exchange for an increase in their payor base and a chance to benefit from cost containment measures. Common risk-sharing methods are prospective payment schedules (PROSPECTIVE PAYMENT SYSTEM), capitation (CAPITATION FEES), diagnosis-related fees (DIAGNOSIS-RELATED GROUPS), and pre-negotiated fees". And also, WHO defines risk-pooling as "the practice of bringing several risks together for insurance purposes in order to balance the consequences of the realization of each individual risk" [4]. Pooling ensures that the risk related to financing health interventions is borne by all the members of the pool and not by each contributor individually. Risk pooling is required because of the large uncertainty in the magnitude and timing of an individual's health care expenditure needs. It implies three redistributive functions: from the rich to the poor, from the healthy to the sick, and from the productive to the unproductive stage of the life cycle [1]. In the concepts of risk-pooling and risk-sharing is important that who pays the costs of the health cares? The results of financial contribution shows in the health system can be analyzed by two approaches: the income approach and the financial burden approach [1] [5].

International research shows that the arguments in favor of risk pooling in health care reflect equity (a transfer of health care resources between individuals) and efficiency (the reduction of individual uncertainty associated with health care needs) considerations [6]. There are essentially four classes of approach to risk pooling [7]: 1) no risk pool, 2) unitary risk pool, 3) fragmented risk pools, 4) integrated risk pools, and below are their definitions: 1) no risk pool: When there is no risk pooling, individuals are responsible for meeting their own health care costs as they arise. In its purest form, this entails patients' meeting user charges as they are incurred, with no subsidy of prices for poorer people and denial of treatment when the patient lacks the financial means to pay. Finally, by definition, any health care system that relies on individuals to make their own financial provision for health care expenditure will fail to address many issues of public health, which are a central concern in many low and lower-income countries. A side effect of pooling is that the risk pool can act as a focus for programs related to population health that the individual or the private insurance market cannot address; 2) unitary risk pool: Under the unitary risk pool, revenue (whether generated by general taxation, social insurance, health care insurance, or user charges) is placed in a single central pool that seeks to cover a chosen package of health care services. Payments are then made to providers in line with patient demands. Under the unitary model, risk pooling must be mandatory, in the sense that rich or healthy citizens cannot opt out of contributing. The mandatory risk pool is one possible policy response to counter the manifest inefficiencies and inequities associated with adverse selection, cream-skimming, and transaction costs; 3) fragmented risk pools. In practice, pursuit of a pure unitary system of risk pooling is usually unfeasible and may be 
undesirable. Although a large unitary risk pool in principle effects complete risk sharing across a nation and minimizes variations in expected expenditure, it brings with it enormous practical difficulties associated with managerial control and coordination. As a result, almost all nations devolve health care purchasing arrangements to smaller organizations, so that the risk pool becomes fragmented; 4) integrated risk pools. The policy response to the efficiency and equity problems brought about by fragmentation has been to develop the notion of integrated risk pools. Under this arrangement, the individual risk pools of the sort discussed above can remain in place, but financial transfers are arranged between pools so that some or all of the variation caused by pure fragmentation is eliminated. In this section we discuss the approaches to integration that have been adopted, under which fragmented risk pools are compensated for the variations in risk to which they are exposed.

The evidences argue that the unitary risk pool is an ideal, but that numerous practical difficulties in making it operational. Instead, it is likely that most health systems will use a system of fragmented pools, and that methods of integration are therefore required [8]. In summary, as risk pooling becomes progressively more integrated, the uncertainty associated with health care expenditure can be reduced. a system of out-of-pocket payments exposes individuals to the greatest level of uncertainty, and on the other hands, Integration risk pooling seeks to reduce these variations, which are eliminated under a truly unitary system [8] (Figure 1).

In the health sector, "Risk pooling and risk financial protection mechanisms" include public/national health insurance, community-based financing, social or private health insurance and pre-payment schemes. Furthermore, Prepayment schemes apply several mechanisms to increase and diversify their risk pool. Prepayment schemes can be for two types of health expenses: high cost and low frequency; and low cost and high frequency. The former involves much greater

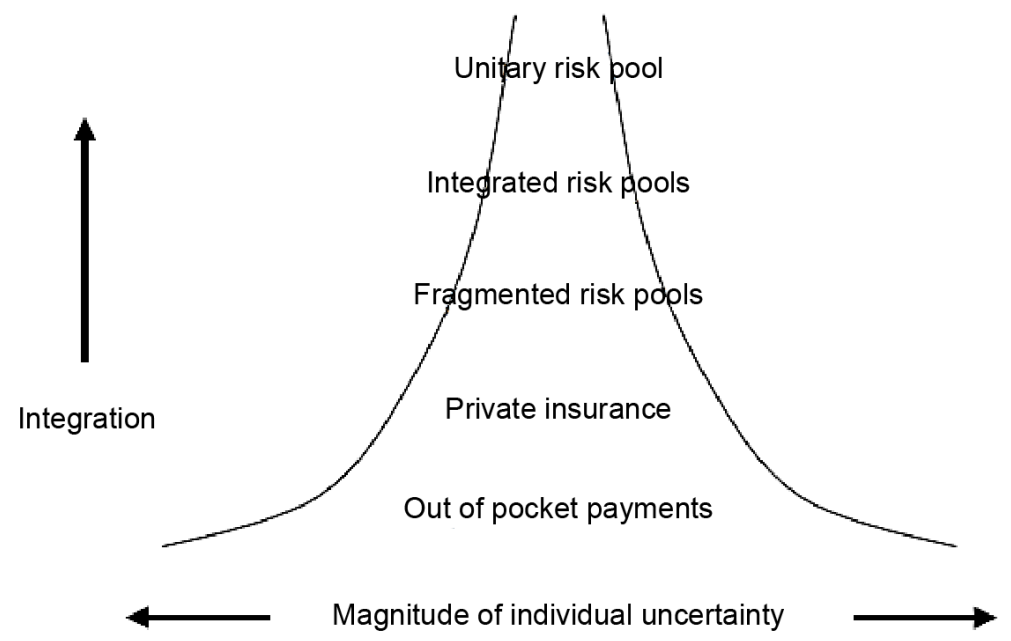

Figure 1. The integration risk-pooling \& risk-sharing pyramid: as integration increases so the degree of uncertainty borne by the individual decreases. 
risk pooling (insurance) than the latter. All health systems collect, manage and allocate resources. In order to achieve the health goals more effectively, sufficient resources must be generated, risks must be pooled effectively and resources must be allocated to services that use health costs more efficiently [9]. In developing countries, the equity argument is particularly acute for two reasons. First, the pattern of burden of disease (still predominantly communicable diseases) is closely related to poverty: the poor (those least able to pay) are the ones most in need to treatment. Second, low absolute levels of income mean that even modest financial contributions can lead to inability to seek treatment or adverse consequences from seeking treatment (such as indebtedness or reduced expenditure on other essential items).

The several studies suggest that, in principle, enormous efficiency and equity gains can be secured by well-designed risk-pooling arrangements. However, numerous practical issues arise in seeking to make the principles of risk pooling operational. This section discusses these issues under eight headings: the institutional framework for risk-pooling arrangements, membership criteria for risk pools, the size of the risk pools, setting capitation payments, variations in the benefit package, retrospective risk sharing, overlapping risk pools, and payment systems and risk sharing. An appropriate health system helps to ease the risk of health financing by providing sufficient resources to improve the health status. In addition, such a risk-based financing mechanism, by revising the barriers that recipients and recipients have in relation to the provision and use of services with them, affects health [1] [8]. An appropriate system for risk sharing in health financing, Helps to improve health by providing adequate resources. In addition, such a risk-based financing mechanism affects health through the modification of incentives for payers and recipients in relation to the provision and use of services to them. The risk-sharing mechanism in the health financing system also influences a range of social goals other than health promotion. This range includes solidarity, financial protection, and accountability. In any case, financing health systems through the collection, accumulation and purchasing functions has a strong impact on fairness in the household's financial contribution to the health system as well as its impact on health and accountability outcomes [1] [10].

The introduction of user charges in many low and lower middle-income countries has been proposed as a strategy to increase revenue. But delayed treatment, catastrophic health expenditures and impoverishment are among the harmful effects attributed to them. To reduce these risks, the mechanisms that allow paying for care in advance or risk pooling have been increasingly advocated. "Risk-sharing \& Risk-pooling mechanisms" include community-based insurance, social or private health insurance and pre-payment schemes. They all share the particularity of involving prospective payments for health care-as opposed to payment at the point of delivery. In all of the schemes funds are collected in advance. Pre-payment schemes are individual forms of health financing 
and offer no opportunity for risk pooling, in contrast to community-based insurance that allows pooling across all beneficiaries of the scheme. Unlike community-based insurance, social health insurance is a compulsory scheme, whose coverage may vary from a specific large group (e.g. formal employees) to the whole population of a country. Social insurance schemes are usually mainly based on payroll contribution, from employers and employees. Increasingly support for such risk protection mechanisms has been voiced on grounds of their theoretical capacity to protect vulnerable people and alleviate financial constraints to accessing health services [3].

This paper has sought to demonstrate that risk-pooling arrangements have important implications for health system behavior and may profoundly affect the purchasing function. Fragmentation of risk pools through devolution poses particular dangers. In developing countries, with high burdens of disease, particularly among the poor, and poor mechanisms for transfer of wealth, there are therefore strong reasons to believe that the trend should be toward larger risk pools than exist at present. Ideally, these would embrace mandatory, universal coverage, in which contributions are either income or community rated and where there is some mechanism for equalization between pools. In practice, the trend has been in the opposite direction, with many countries devolving health care arrangements to local levels and not implementing any risk-pool integration [8].

In 2018 [7], according WHO's method for the classification of risk-sharing in health care financing, WHO's data (authors, 2018) showed that between 2000-2014 the degree of risk-sharing in low-income countries (from 1.58 to 2.08; of the total 6 points Likert) is low risk-sharing and in lower middle-income countries (from 2.47 to 2.86 ) is medium risk-sharing. This rapidly shift in these income countries groups was coincided (1995-2014) with increasing general government expenditure on health (GGHE) as a share of total health expenditure (THE) in low-income countries(from $33.6 \%$ to $41.2 \%$ ) and lower middle-income countries (from $34.9 \%$ to $36.2 \%$ ), reducing in Private expenditure on health as a percentage of total expenditure on health in low-income countries (from $66.4 \%$ to $58.8 \%$ ) and lower middle-income countries (from $65.1 \%$ to $63.8 \%$ ), reducing Out-of-pocket expenditure as a percentage of private expenditure in low-income countries (from $80.8 \%$ to $65.5 \%$ ) and lower middle-income countries (from $89.4 \%$ to $87.5 \%$ ). in addition, in time period 1995-2014, share of External resources for health as a percentage of total expenditure on health in low-income countries (from $13.1 \%$ to $28.3 \%$ ) and lower middle-income countries (from $1.8 \%$ to $3.3 \%$ ) had been high increased [7].

Improved health status in low and lower middle-income countries could considerably improve wellbeing and possibly promote economic growth. The last decade has seen a surge in field experiments designed to understand the barriers that households and governments face in investing in health and how these barriers can be overcome, and to assess the impacts of subsequent health gains. In many more studies have been examined the determinants of individual health 
behavior, on the side of consumers as well as among providers of health products and services. The empirical evidence presented above suggests that the exact relationship between health care spending and health outcomes is not clear, especially at the macro level. While some studies have shown significant positive or negative impact of health care spending on health outcomes [11].

Improvement in human capital has been identified as a critical catalyst to economic growth and development in the macroeconomic literature. Grossman's human capital model suggests that quality health significantly influences human capital development through the additional working time and utility derived from good health. Good health does not only improve individuals' consumption and production in the short run, but also improves returns from investments in productive activities in the long run. Adequate and efficient health related spending is widely considered as inevitable in the improvement of health status. At the macro level, investment in health workforce and infrastructure is expected to improve health conditions and hence human capital of the population. However, in sub-Saharan Africa (SSA) and other developing regions where resources are relatively scarce, health expenditure has received less attention in government budgets [11].

According deferent studies showed that many factors which affect the promotions of health care in low and lower middle-income countries such as cost-sharing in health care expenditure, education attainments, economic development, income level. Health care expenditure has been low over the years in developing regions of the world. A majority of countries in low and lower middle countries, especially sub-Saharan Africa (SSA), rely on donor grants and loans to finance health care. Such expenditures are not only unsustainable but also inadequate considering the enormous health care burden in the region. in recent studies [7] [12] [13] indicate that both government and private spending on health care significantly improve infant, under-five, and maternal mortality in Middle East and North Africa (MENA) Region [10].

The results show that health care expenditure significantly influences health status through improving life expectancy at birth, reducing death and infant mortality rates [13]. And also, Medical facilities financing management can have a significant impact on reducing costs and increase the quality of health cares. Therefore, it is essential that the selection of health financing arrangements in an objective manner that they will be classified according to their importance in better financial risk protection in health systems [14]. Both public and private health care spending showed strong positive association with health status even through public health care spending (with greater degree of risk-sharing) had relatively higher impact on health outcomes. And also, the relationships are causal in nature. Specifically, a percentage increase in per capita government expenditures reduces the infant mortality rate by 8.6 - 9.5 deaths per 1000 live births $(\mathrm{p}<0.01)$, the under-five mortality by $10.3-12.1$ under-five deaths per 1000 live births $(\mathrm{p}<0.01)$, and maternal mortality by $26.0-26.3$ deaths per 100,000 live births $(\mathrm{p}<0.01)$. Similarly, a percentage increase in the log per cap- 
ita private expenditures reduces the infant mortality rate by $7.2-8.1$ deaths per 1000 live births $(\mathrm{p}<0.01)$, under-five mortality rate by $9.5-9.8$ deaths per 1000 live births $(\mathrm{p}<0.01)$, and the maternal mortality rate by $25.8-25.9$ deaths per 100,000 live births $(\mathrm{p}<0.01)$. Anyanwu and Erhijakpar (2007) used data from 47 African countries between 1999 and 2004 and provided econometric evidence that health expenditures have a statistically significant effect on infant mortality and under-five mortality. Gottret and Scieber (2006) conducted a study of 81 low and middle income countries and found that a 10 percent increase in government health expenditure has a larger impact in reducing under-five mortality and maternal mortality than a 10 percent increase in education, roads and sanitation [13].

Furthermore, different studies confirm that risk pooling and prepayment, no matter how small scale, improve financial protection (directly, by preventing impoverishment, or indirectly, by ensuring access to health care, thereby improving health and social opportunities) for the populations they serve. When health financing systems are aggressive (based on high greater risk pooling/risk sharing such as public resources and pre-payments), financial health risk is less for households and better risk sharing, equitable and efficient. On the other hand, regressive health financing systems that rely on direct payments especial OOPs, have no risk pooling/risk sharing and greeter financial health risks for households, inequitable and inefficient [1] [7]. Therefore, adopting and operating financing policies based on greeter risk pooling/sharing such as prepayments schemes (public health coverage) are recommended to all countries, especially in low and lower-middle income countries. It means risk sharing/pooling plays a key role in all financing systems to achieving effectiveness and efficiency and moving towards Universal Health Care Coverage (UHCC) [1] [7].

Finally, health financing policies through pre-payment schemes and public resources with higher risk sharing (health pooled funds), such as universal health coverage in high-income countries include United Kingdom(90\%), Germany $(89 \%)$, Sweden $(85 \%)$, and in middle-income countries such as Chile (66\%), South Korea (65\%), Brazil (69\%), Mexico (52\%), Thailand (84\%), Rwan$\mathrm{da}$, and Turkey, have a progressive financing system and lower financial risk burden and lower health catastrophic cost for people [1]; and in low and lower middle income countries, mostly private financing policies, especially OOPs, have most regressive effect, maximum financial risk burden and more impoverishment, for community. Therefore, health financing reforms with better risk pooling (such as pre-payment schemes, community based financing, social health insurance) need to be seen as a key strategy in achieving UHC and reducing financial barriers to health care, higher utilization but lower out-of-pocket expenditure, in low and lower middle income countries.

\section{Financial Support and Sponsorship}

This study was approved and scientifically supported by the Tarbiat Modarres 
University (TMU), Tehran, Iran.

\section{Conflict of Interests}

The authors declare that there is no conflict of interests.

\section{Biography}

Ahangar A. (PhD Candidate in Health Economics, School of Economics and Management); Ahmadi A.A. (Corresponding Author, Assistant Professor, PhD, Economic Research Center (ERC); Mozayani A.H. (Associate Professor, PhD, Economic Research Center (ERC); Faraji Dizaji S. (Assistant Professor, PhD, School of Economics and Management), Tarbiat Modarres University (TMU), Tehran, Iran

\section{References}

[1] Ahangar, A., Ahmadi, A.M., Mozayani, A.H. and Faraji Dizaji, S. (2018) Transition of Health Financing Models and Cost Risk Sharing in the Health Sector: A Systematic Review. Iranian Red Crescent Medical Journal. (In Publishing)

[2] Shigeoka, H. (2013) The Effect of Patient Cost Sharing on Utilization, Health, and Risk Protection. National Bureau of Economic Research, Working Paper Series, No. 19726.

[3] Preker, A.S. and Carrin, G. (2004) Health Financing for Poor People Resource Mobilization and Risk Sharing. The World Bank, The International Bank for Reconstruction and Development, Washington DC.

[4] World Health Organization (2000) The World Health Report 2000. Health Systems: Improving Performance. World Health Organization, Geneva.

[5] Murray, C.J.L., Xu, K., Evans, D., Klavus, J., Kawabata, K., Hanvoravongchai, P., et al. (2003) Assessing the Distribution of Household Financial Contributions to the Health System: Concepts and empirical Application. In: Murray, C.J.L. and Evans, D.B., Eds., Health System Performance Assessment (Debates, Methods and Empiricism), World Health Organization, Geneva, 565-572.

[6] Ahmadi, A.M., Maher, A. and Shokri, J.A. (2008) Survey of Different Approaches to Health System Financing in the Selected Countries during the Period 1998-2004 and Introducing New Financing Mechanisms for Iran. The Economic Research, 8, 149-155.

[7] Ahangar, A., Ahmadi, A.M., Mozayani, A.H. and Faraji Dizaji, S. (2018) The Assessment of Risk-Sharing in the Financing of the Health Sector and Health Outcomes; a Comparative Study across WHO's Regions. PhD. Dissertation, Tarbiat Modarres University (TMU), Tehran.

[8] Smith, P.C. and Witter, S.N. (2004) Resource Allocation and Purchasing in Health: Value for Money, Reaching the, Poor Risk Pooling in Health Care Finance. World Bank, Centre for Health Economics, Washington DC.

[9] Savedoff, D.W., Carrin, G., Kawabata, K. and Mechbal, A. (2003) Monitoring the Health Financing Function. In: Murray, C.J.L. and Evans, D.B., Eds., Health System Performance Assessment (Debates, Methods and Empiricism), World Health Organization, Geneva, 211-219.

[10] Gallet, C.A. and Doucouliagos, H. (2017) The Impact of Healthcare Spending on Health Outcomes: A Metaregression Analysis. Social Science \& Medicine, 179, 9-17. 
https://doi.org/10.1016/j.socscimed.2017.02.024

[11] Anyanwu, C.J. and Erhijakpor, E.O.A. (2007) Health Expenditures and Health Outcomes in Africa. African Development Bank Economic Research Working Paper No. 91. http://www.afdb.org/fileadmin/uploads/afdb/Documents/Knowledge/

[12] Novignon, J., et al. (2012) The Effects of Public and Private Health Care Expenditure on Health Status in Sub-Saharan Africa: New Evidence from Panel Data Analysis. Health Economics Review, 2, 22. https://doi.org/10.1186/2191-1991-2-22

[13] Akinci, F., Hamidi, S., Suvankulov, F. and Akhmedjonov, A. (2014) Examining the Impact of Health Care Expenditures on Health Outcomes in the Middle East and North Africa (MENA) Region. Journal of Health Care Finance, 41, 1-23.

http://www.HealthFinanceJournal.com

[14] Safarani, S., Khatami Firouzabadi, S.M.A. and Ahangar, A. (2017) Supplier Selection for Serum and Syringe Using Multi-Criteria Decision-Making Methods ELECTRE1, TOPSIS and Compared Them with VIKOR. Payavard Salamat, 11, 380-390. 